\title{
Inkjet-printed, functional heterolayers of ZnO@CuO for stoma pouch monitoring
}

Alvaro Ortiz Perez ${ }^{1}$, Haitao Gao ${ }^{1}$, Xuemeng Lyu ${ }^{1}$, Jürgen Wöllenstein ${ }^{1,2}$, Vera Kallfaß3 , Jordi Fonollosa ${ }^{4,5}$, Stefan Palzer ${ }^{6, *}$

${ }^{1}$ Laboratory for Gas Sensors, Department of Microsystems Engineering - IMTEK, University of Freiburg, Freiburg, Germany

${ }^{2}$ Fraunhofer Institute for Physical Measurement Techniques (IPM), Freiburg, Germany

${ }^{3}$ Steinbeis Transfer Center, Social Planning, Qualification and Innovation, Meersburg, Germany ${ }^{4}$ B2SLab, Departament d'Enginyeria de Sistemes, Automàtica i Informàtica Industrial, Universitat Politècnica de Catalunya, Barcelona, Spain

${ }^{5}$ Institut de Recerca Pediàtrica Hospital Sant Joan de Déu, Esplugues de Llobregat, Barcelona, Spain ${ }^{6}$ Escuela Politécnica Superior, Universidad Autónoma de Madrid, Madrid, Spain

*Corresponding author e-mail address: stefan.palzer@uam.es; Telephone number: +91 4975720

\begin{abstract}
Many bowel cancer patients are in need of an artificial stoma as part of their surgical treatment, and associated post-surgical odors caused by leaking stoma pouches may lead to social isolation, which is why inconspicuous monitoring of this situation is important for affected persons. The integration of micro- and nanotechnology may offer low-cost, low-power consuming and small solutions to this challenge. To this end, we present an inkjet-printed, heterostructured gas sensor that has been built by incorporating nanosized p-type semiconducting $\mathrm{CuO}$ in a porous n-type $\mathrm{ZnO}$ matrix. The functional
\end{abstract}


layer is fabricated using a combination of a colloidal suspension and sol-gel approach optimized for inkjet printing thus offering an industry-ready method for integration of nanomaterials in microelectromechanical systems (MEMS) structures. Using a thermal modulation scheme we enhance the information content and classify different events. We demonstrate that a simple MEMS device using a novel hetero-nanomaterial may be used to reliably identify situations where stoma pouch content escapes.

\section{Keywords}

$\mathrm{CuO}, \mathrm{ZnO}$, hybrid functional materials, colloidal suspension, inkjet printing, additive manufacturing

\section{Introduction}

About $18 \%$ of new cancer diagnoses are associated with the digestive system [1]. If surgical treatment is necessary it may be accompanied by installation of artificial exits, so-called ostomies. Even though this technique is well-established, it is still cumbersome to maintain a sealed stoma pouch on a regular basis, which causes frequent leaks and associated bad odors. So apart from the oftentimes dire outlook for patients, a secondary effect associated with bad odor upon escape of pouch content in combination with a decreasing sense of smell limits the social engagement, ultimately leading to a deterioration of social participation [2]. In fact, a recent study found that stoma pouch leak problems are among the top research priorities [3]. This situation gives rise to the need for unsuspicious monitoring of the state of the stoma pouch. We propose to tackle this using a hybrid nanomaterial integrated into a microsystem to detect leaks in the pouch, which would allow for a low-cost, mass deployable system. Previously, we showed that a hotplate-based miniature system with an autonomous operation time of several days can be successfully fabricated and used [4]. 
Here, the central building block of the gas sensing micro device is a hybrid layer made of two types of functional nanomaterials. The chemical composition of human faeces is highly complex and the bad odour emitted is a blend of a plethora of sulphur and amines containing molecules, including but not limited to hydrogen sulfide $\left(\mathrm{H}_{2} \mathrm{~S}\right)$, methylmercaptan $\left(\mathrm{CH}_{3} \mathrm{SH}\right)$, triethylamine/trimethylamine $\left(\left(\mathrm{C}_{2} \mathrm{H}_{5}\right)_{3} \mathrm{~N}\right)$, and ammonia $\left(\mathrm{NH}_{3}\right)$ [5]. Because of the properties in selective trace gas detection [6]-[10] towards sulphur containing molecules we use copper(II)oxide as additive in a zinc oxide ( $\mathrm{ZnO}$ ) layer, which is among the best researched materials for gas sensing applications [11]-[15]. Using thermal modulation protocols the reaction between $\mathrm{CuO}$ and sulphur containing molecules may be tailored to achieve highly selective trace gas sensing components [16]. Using inkjet printing technology we produce a heterolayer of $p$-type semiconducting copper(II)oxide ( $\mathrm{CuO}$ ) nanospheres integrated into porous $\mathrm{n}$ type zinc oxide, thus forming $p$-n junctions at the interface between both types of materials. In the past the combination of these materials has been fabricated in numerous ways and its gas sensitive response has been investigated. In 1990 Nakamura et. al. [17] have showed increasing selectivity of $\mathrm{ZnO} / \mathrm{CuO}$ heterojunctions and in recent years nano-sized matrices haven been fabricated and researched. Among the realizations are core shell CuO-ZnO nanocomposites [18], nanoflakes [19], Al doped CuO-ZnO structures [20], free-standing $\mathrm{ZnO}-\mathrm{CuO}$ nanowire arrays [21], CuO decorated $\mathrm{ZnO}$ hierarchical structures [22], and CuO-ZnO nanorods [23]. CuO-ZnO heterojunctions have also been obtained using a sol-gel approach [24], sputtering [25], hydrothermal process [26] as well as derived from powders [27] or a combination of sputtering and chemical vapor deposition [28]. Also, the incorporation of a $\mathrm{ZnO} / \mathrm{CuO}$ heterostructure on a hotplate also has been achieved recently using sputtering of brass [29]. Nonetheless, so far the potential of inkjet printing of colloidal suspensions for the deposition of heterolayers on arbitrary platforms has been explored less frequently. Some advantages of using colloidal suspensions are the facile control of size and shape of the nanoparticles [10], the precise control of layer thickness [30], [31], as well as using a flexible, industry ready deposition technique that may be used to deposit suspensions on arbitrary substrates [32], [33]. 
In this contribution both materials are produced using a scalable, wet-chemistry synthesis route to obtain a printable ink containing both nanomaterials. By mixing inks made up of colloidal CuO nanospheres and a sol-gel solution for $\mathrm{ZnO}$, the $\mathrm{CuO}$ nanoparticles are incorporated into a matrix of porous $\mathrm{ZnO}$ as depicted in Figure 1.

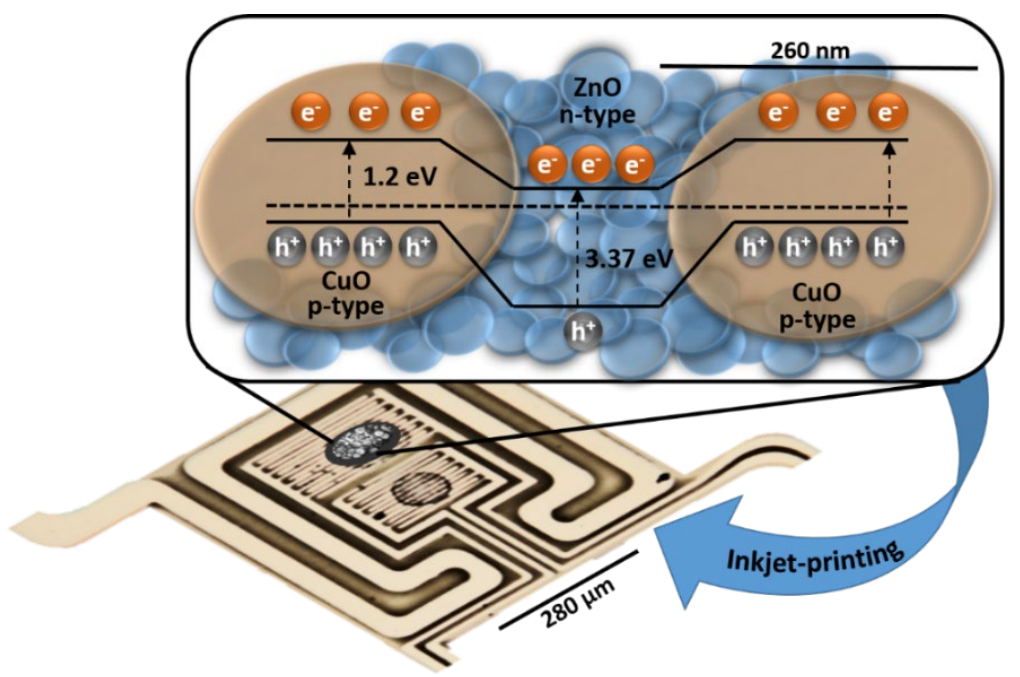

Fig. 1: Using inkjet printing technology a novel type of hybrid nanomaterial is deposited onto lowpower consuming MEMS structure that enables electronic read-out of the layer as well as thermal control.

Both inks feature a low solid content, such that a scalable, precise control of the final layer's thickness becomes possible [30]. Using the inkjet printing technology offers a way to bridge the gap between nanoscience and MEMS and integrate hybrid nanomaterials in a flexible, reproducible fashion. Thermal control and electronic read-out of the final functional layer is achieved using a micromachined, lowpower consuming suspended hotplate device featuring a heating structure and two interdigitated electrode structures [34], [35]. Using this simple device in combination with a temperature modulation scheme different odor scenarios are classified.

\section{Experimental}

\section{$\underline{\text { Ink preparation }}$}


As a first step we prepare cuprous oxide $\left(\mathrm{Cu}_{2} \mathrm{O}\right)$ nano-particles according to our previously described synthesis method [30]. $0.4832 \mathrm{~g}$ trihydrated copper nitrate $\mathrm{Cu}\left(\mathrm{NO}_{3}\right)_{2} \bullet 3 \mathrm{H}_{2} \mathrm{O}$ is dissolved in $40 \mathrm{ml}$ water $\left(\mathrm{H}_{2} \mathrm{O}\right)$ and then kept stirring for about $30 \mathrm{~min}$ at $250 \mathrm{rpm}$. Meanwhile, $0.4 \mathrm{~g}$ polyvinylpyrrolidone (PVP, average $\mathrm{MW}=55,000$ ) is dissolved in $60 \mathrm{ml}$ water. After complete dissolution the PVP solution is added dropwise to the $\mathrm{Cu}\left(\mathrm{NO}_{3}\right)_{2}$ solution and the reaction mixture is stirred for $2 \mathrm{~h}$. Then, $90.6 \mu \mathrm{l}$ of hydrazine $\left(\mathrm{N}_{2} \mathrm{H}_{4}\right)$ solution (35\% $\mathrm{N}_{2} \mathrm{H}_{4}$ in water) is added to the reaction mixture drop-wisely. The color of the solution changes into orange immediately after the introduction of $\mathrm{N}_{2} \mathrm{H}_{4}$, which indicates the production of $\mathrm{Cu}_{2} \mathrm{O}$ nanoparticles. The resulting solution is kept stirring for $20 \mathrm{~min}$ at room temperature and then washed with $\mathrm{H}_{2} \mathrm{O}$ and ethanol (EtOH) several times by centrifugation and redispersion (Multifuge $3 \mathrm{SR}$, Heraeus: $900 \mathrm{~g}, 45 \mathrm{~min}, 21^{\circ} \mathrm{C}$ ). In a final step the $\mathrm{Cu}_{2} \mathrm{O}$ nanoparticles are redispersed in $6 \mathrm{ml} \mathrm{EtOH}$ and stowed away in the dark until further use. Prior to mixing with the $\mathrm{ZnO}$ ink, the $\mathrm{Cu}_{2} \mathrm{O}$ ink is further diluted using $1 \mathrm{ml}$ of above ink diluted into $5 \mathrm{ml}$ ethanol in order to avoid agglomeration. Secondly, $\mathrm{ZnO}$ ink is prepared based on the recipe presented in [36]. $0.5 \mathrm{mmol}$ zinc acetate dehydrate $\left(\mathrm{Zn}(\mathrm{OAc})_{2 \cdot 2}{ }_{2} \mathrm{H} 2 \mathrm{O}\right)$ (Sigma-Aldrich) and $1.25 \mathrm{mmol} \mathrm{NaOH}$ (Sigma-Aldrich) are dissolved in $100 \mathrm{ml}$ 2-propanol under stirring at $50^{\circ} \mathrm{C}$. Then $0.1 \mathrm{~g}$ PVP ( $\mathrm{Mw}=40,000$, Sigma-Aldrich) is added to the solution under stirring. The above solution is brought into an ultrasonic bath for two hours. The final product is washed using water and $\mathrm{EtOH}$ for three times. The white $\mathrm{ZnO}$ particles are dispersed in $3 \mathrm{ml}$ ethanol, leading to a stable ink. Finally, $1 \mathrm{ml} \mathrm{ZnO}$ ink is mixed into $2 \mathrm{ml} \mathrm{Cu}_{2} \mathrm{O}$ ink under stirring for two hours. The original orange $\mathrm{Cu}_{2} \mathrm{O}$ ink becomes light-colored due to the addition of $\mathrm{ZnO}$ ink. The complete process is schematically displayed in Figure 2. 


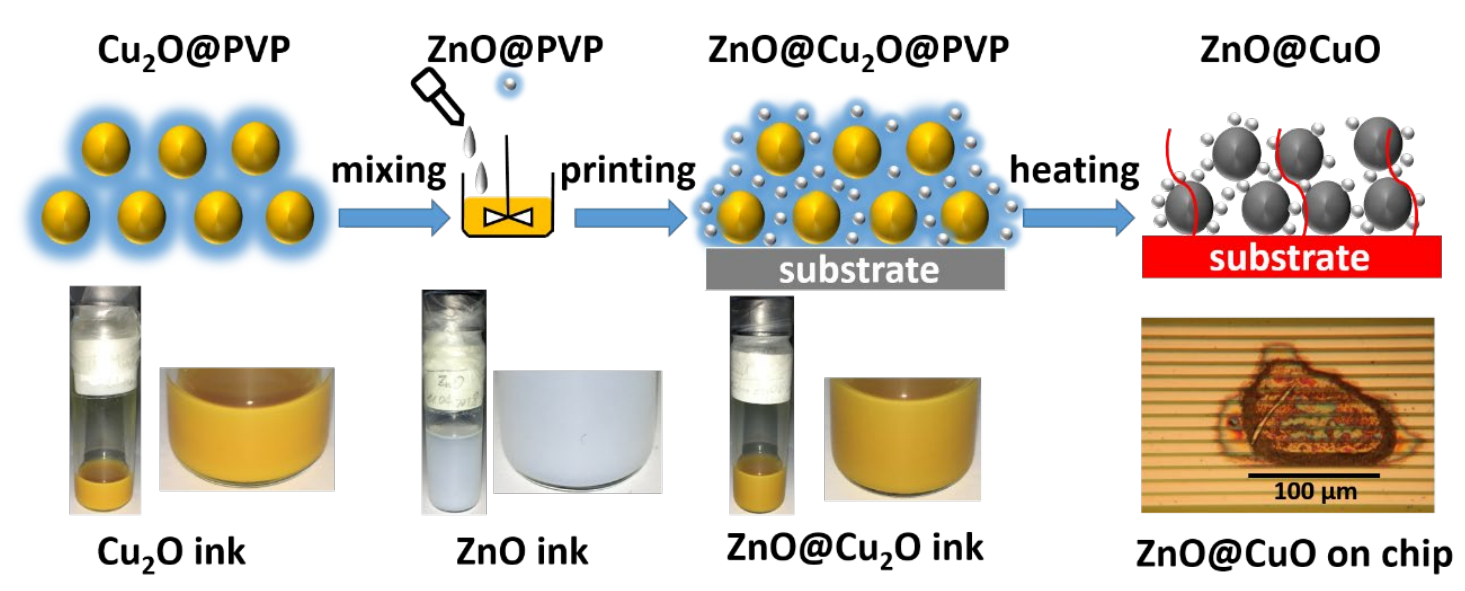

Figure 2. The process for preparation of ZnO@CuO composite functional layer starts from individual $\mathrm{ZnO}$ and $\mathrm{Cu}_{2} \mathrm{O}$ inks. The $\mathrm{ZnO} @ \mathrm{CuO}$ composite layers are produced by mixing the two single inks, depositing the resulting mixture onto interdigitated electrode structures and subsequently burning off the superficial polymer, which simultaneously promotes the conversion of $\mathrm{Cu}_{2} \mathrm{O}$ to $\mathrm{CuO}$.

\section{$\underline{\text { Integrated microsystem }}$}

We deposit the composite nano-material onto a hotplate structure that has been optimized for the employed DIMATIX-DMP 2831 inkjet printing system [34]. A two-array hotplate chip is used as substrate, which contains two interdigitated electrode (IDE) structures with areas of (280 x 135) $\mu \mathrm{m}^{2}$ with 7 pairs of electrodes and $(220 \times 115) \mu \mathrm{m}^{2}$ with 6 pairs of electrodes, respectively. The inter electrode spacing as well as width of each electrode are $5 \mu \mathrm{m}$. A single heater provides control over the temperature of the two IDE structures to reach the target, simultaneously. To deposit the ink, the print head cartridge is filled with $1.5 \mathrm{ml}$ of the ink and a voltage of $29 \mathrm{~V}$ is applied to the piezoelectric nozzle of the inkjet printer to release an ink drop. The $\mathrm{ZnO} @ \mathrm{Cu}_{2} \mathrm{O}$ layer is produced in a pattern of $3 \mathrm{x}$ 1 dots with a set spacing of $15 \mu \mathrm{m}$ between each dot. This deposition is repeated 3 times at the same area. Finally, the solvent as well as the PVP are removed via heating of the layer to $400^{\circ} \mathrm{C}$ for 4 hours in air, which also converts $\mathrm{Cu}_{2} \mathrm{O}$ to $\mathrm{CuO}$ while maintaining the nanoparticle shape [10]. We have performed scanning electron microscopy (SEM) analysis and x-ray powder diffraction spectroscopy (XRD) on the $\mathrm{CuO}$ and $\mathrm{ZnO}$ material after the thermal treatment. SEM has been performed using a 
Hitachi SU-70, and XRD on CuO has been taken from our previous work [30], while XRD for ZnO has been carried out using a STOE Stadi P driven by Cu K- $\alpha$ radiation.

\section{Laboratory test}

Using a custom build measurement apparatus leaning on the one presented in [37], we exposed the sensing chip to different odor scenarios. We place samples of a stoma pouch, urine and water into a $500 \mathrm{ml}$ polypropylene co-polymer (PPCO) container each. The sample bottles are filled with $300 \mathrm{ml}$ in the case of water, approximately $125 \mathrm{ml}$ for urine and between 100 and $250 \mathrm{ml}$ for the stoma pouch content, depending on sample consistency. Each measurement is performed with a new stoma sample of a different volunteer donor and collected and used at the same day to ensure odor sampling. The air above each container is sucked through a pipe system and directed to the sensing chip located in an airtight box with a volume of $2.3 \mathrm{I}$. The setup is schematically depicted in Figure 3 .

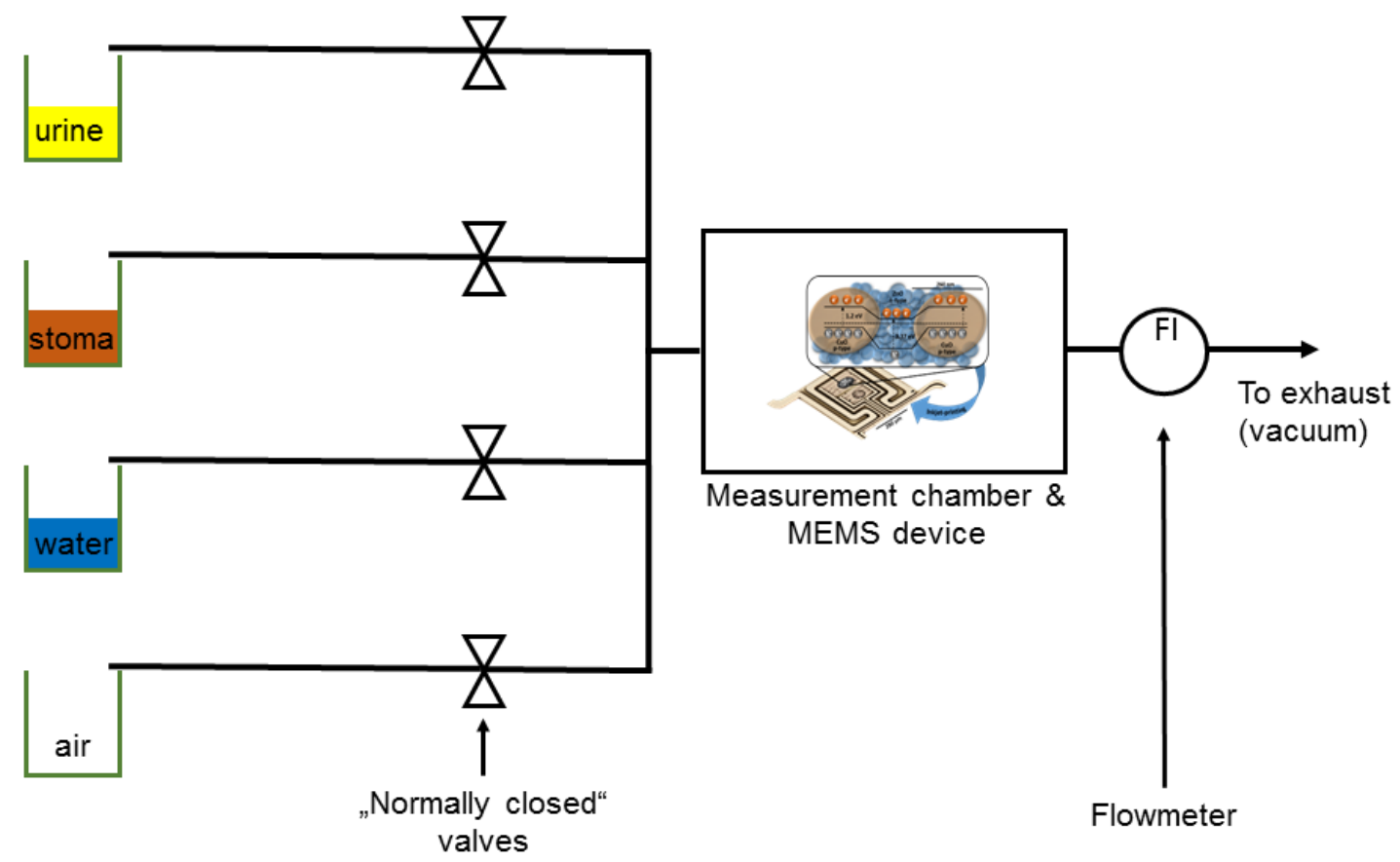

Figure 3. Schematic drawing of the experimental setup to present different gas matrices to the MEMS device. Using 4 different odour channels the gas composition inside the measurement chamber is 
controlled by sucking the air above the respective odour reservoirs and directing the flow to the sensor unit. Using a flowmeter a constant flow is ensured.

Each odor channel is equipped with a valve, such that only one of the channels actually conducts sample air to the sensor, and a flow meter to control a continuous flow of one liter per minute. Automatic control of the valves allows for annotating each situation. The sensor is exposed to each situation for 20 minutes with a clean air step between every situation consisting of exposure to 20 minutes of laboratory air as reference scenario. We have performed a total of 74 measurements over a 5-day time span using a layer temperature of $330^{\circ} \mathrm{C}$.

\section{Calibration model}

We have built a calibration model using the acquired dataset to show the ability of the developed sensor technology to detect odors coming from stoma pouches in a reliable manner. Specifically, we have followed the traditional steps for chemical gas sensor data [38], [39]. First, we applied a preprocessing step consisting on a linear interpolation to correct for missing data points that the time signals may contain. Next, for each sensor exposure, we have extracted six different features. In particular, we computed four steady-state features: the maximum value $\left(f_{1}\right)$, the minimum value $\left(f_{2}\right)$, the signal amplitude $\left(f_{3}=f_{1}-f_{2}\right)$, and the relative amplitude $\left(f_{3} / f_{1}\right)$. We also considered two features that account for the sensor dynamics during the increasing transient of the sensor response. In particular, we used the exponential moving average (ema) that has already been applied in chemical sensing [40]. The output of filtered sensor signals depends on the smoothing parameter $\alpha$. We have extracted the features from the maximum values after applying the ema filter to the sensor signals, for $\alpha=0.1\left(f_{5}\right)$ and $\alpha=0.5\left(f_{6}\right)$. These features aim at incorporating the information contained in the dynamics of the sensors into the model. Therefore, each experiment is represented by an aggregate of 6 features. We have built classification models based on Linear Discriminant Analysis (LDA) using a double-cross 
validation approach to train and test the model. First, $20 \%$ of the samples were set aside for evaluating the performance of the model. The model was built with the remaining $80 \%$ of the samples. In calibration, the features were normalized to zero mean and unit variance. The process was repeated five times until all the samples were left for test once. The reported performance is the mean of the performance after the five repetitions.

\section{Results and Discussion}

\section{Material characterization}

The result of SEM analysis of the individual nano materials is shown in Figure 4. The $\mathrm{Cu}_{2} \mathrm{O}$ ink results in spherical CuO nanoparticles with a diameter of about $260 \mathrm{~nm}$ after thermal treatment. The phase transition from $\mathrm{Cu}_{2} \mathrm{O}$ to $\mathrm{CuO}$ does not change the shape or size of the nanoparticles and XRD reveals a complete transition from the $\mathrm{Cu}_{2} \mathrm{O}$ phase to pure $\mathrm{CuO}$. The $\mathrm{ZnO}$ ink results in a thin, nanoporous layer, which is expected to exhibit high sensitivity. XRD analysis shows that pure $\mathrm{ZnO}$ is obtained via this process.
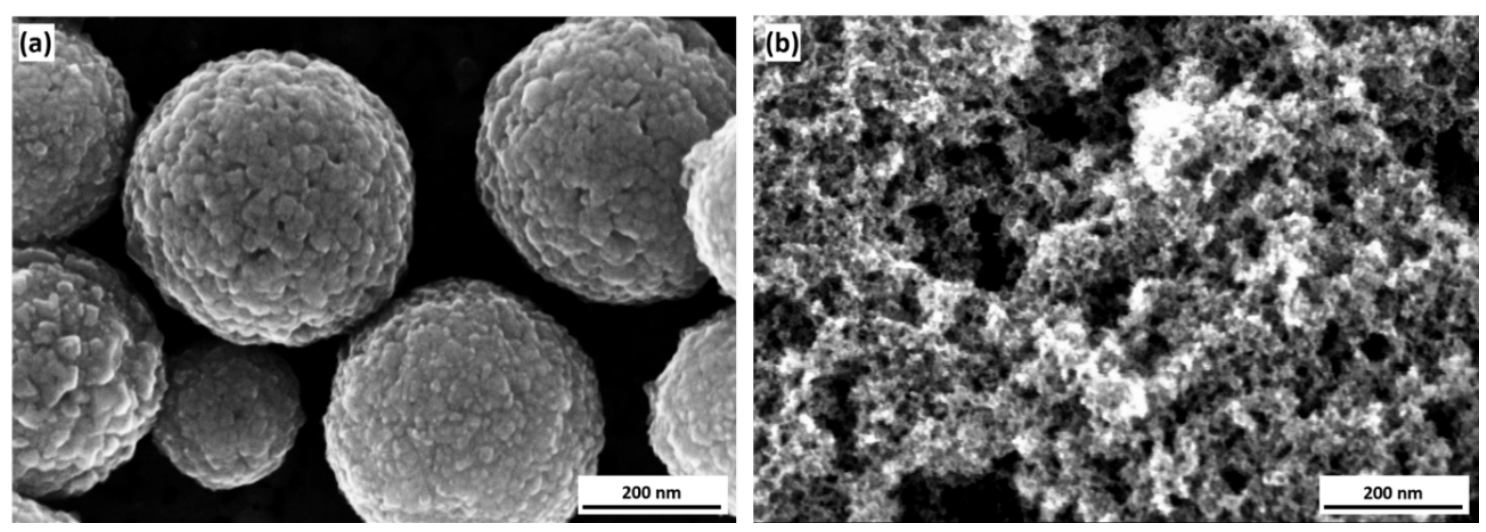

Fig. 4: (a) The colloidal suspension leads to a layer of $\mathrm{CuO}$ nanospheres with low polydispersitivity index. Analysis of SEM images indicate an average size of $260 \mathrm{~nm}$. (b) The ZnO ink leads to a porous $\mathrm{ZnO}$ nanolayer which serves as a matrix into which the $\mathrm{CuO}$ nanospheres are embedded. 
The SEM analysis of the combined $\mathrm{ZnO}$ and $\mathrm{CuO}$ inks is shown in Figure 5 and reveals that the resulting layer is composed of a porous $\mathrm{ZnO}$ into which the $\mathrm{CuO}$ nanoparticle are embedded, forming a p-n junction at their interface. The technique may be further expanded to add different additive or dopants to a porous functional layer. E.g. the incorporation of well-defined, nanoscaled catalyst into a gas sensing layers using an industry ready, scalable inkjet process becomes possible.
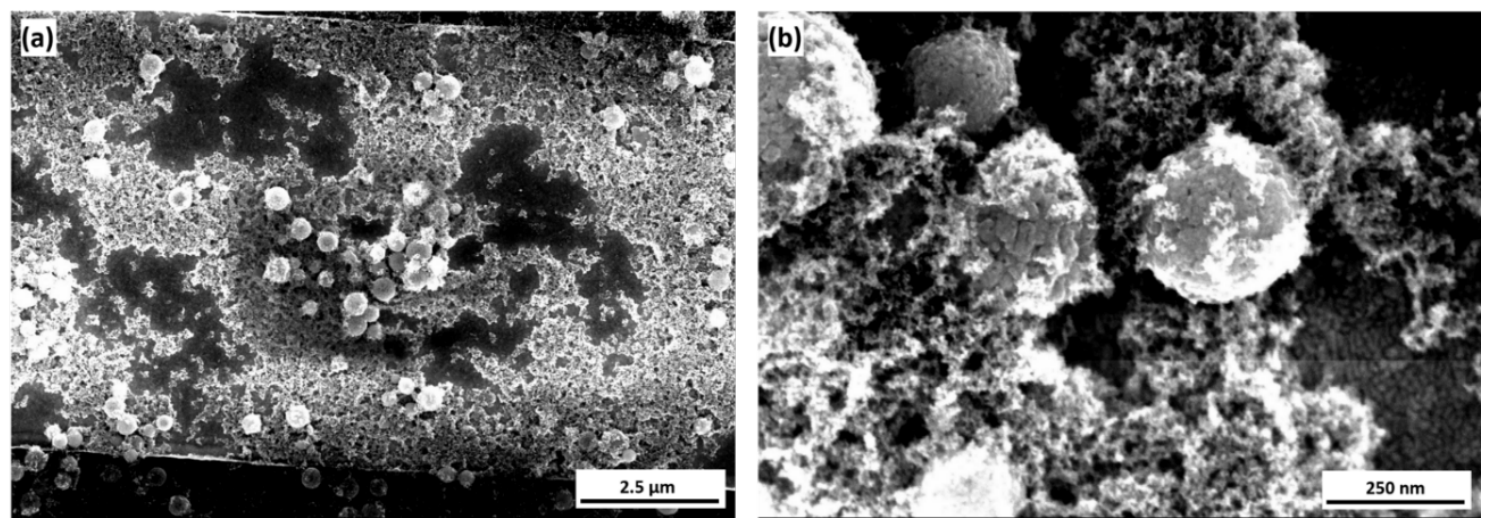

Fig. 5: (a) Overview of the area between two electrodes of the interdigitated electrode structure covered with the CuO@ZnO heteromaterial. The low density of $\mathrm{CuO}$ nanoparticles in combination with the PVP shell help to prevent the formation of clusters. (b) A 80,000 fold magnification shows how the CuO nanosphere are embedded into the porous $\mathrm{ZnO}$ matrix.

The XRD analysis of $\mathrm{CuO}$ and $\mathrm{ZnO}$ is shown in the Figure 6. The results on $\mathrm{CuO}$ indicate the complete phase transformation from $\mathrm{Cu}_{2} \mathrm{O}$ to $\mathrm{CuO}$ taking place after the PVP polymer is burn off in the air. The result in on $\mathrm{ZnO}$ confirms the production of a pure $\mathrm{ZnO}$ layer. The width of the reflection peaks of $\mathrm{ZnO}$ confirm nano-particles composed of crystallites with an estimated average size of $3.4 \mathrm{~nm}$ according to the Debye-Scherer equation [41]. 

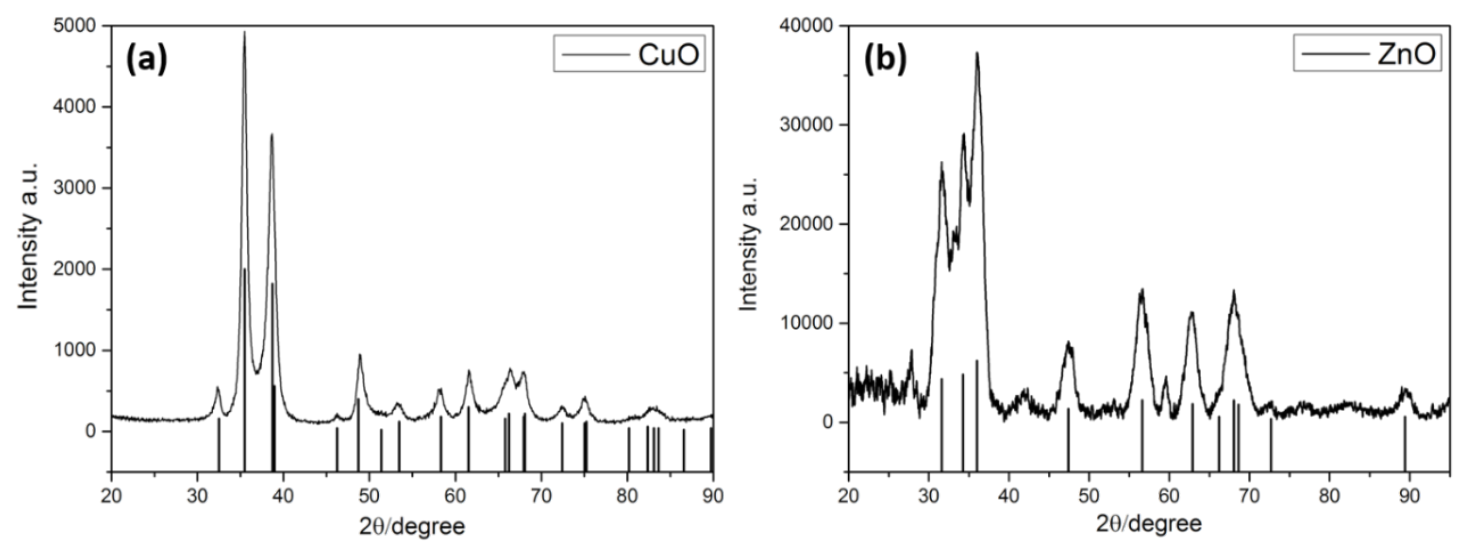

Fig. 6: (a) X-ray powder diffraction measurements confirms the phase transformation from $\mathrm{Cu}_{2} \mathrm{O}$ to CuO after PVP is burned off in the air. The data is taken from [30]. (b) XRD of the ZnO layer also confirms that the product is made of pure $\mathrm{ZnO}$ nanoparticles but including a minor unknown impurity (JCPDS card No. 36-1451)

\section{Odor recognition using ZnO@CuO}

Figure 7 clearly shows the sensitivity of the developed sensor technology to odours coming from a stoma pouch. However, sensor drift and cross-sensitivities to uncontrolled environmental conditions and other volatiles still represent a major hurdle in chemical sensing [42], [43], especially when operating in applications beyond the laboratory [44], [45]. As a result, the definition of a threshold that operates over the sensor amplitude would not provide a reliable calibration model. For example, Figure 7 shows sensor responses under the same stimulus in two consecutive days. A calibration model that goes beyond the mere sensitivity test is therefore necessary. 


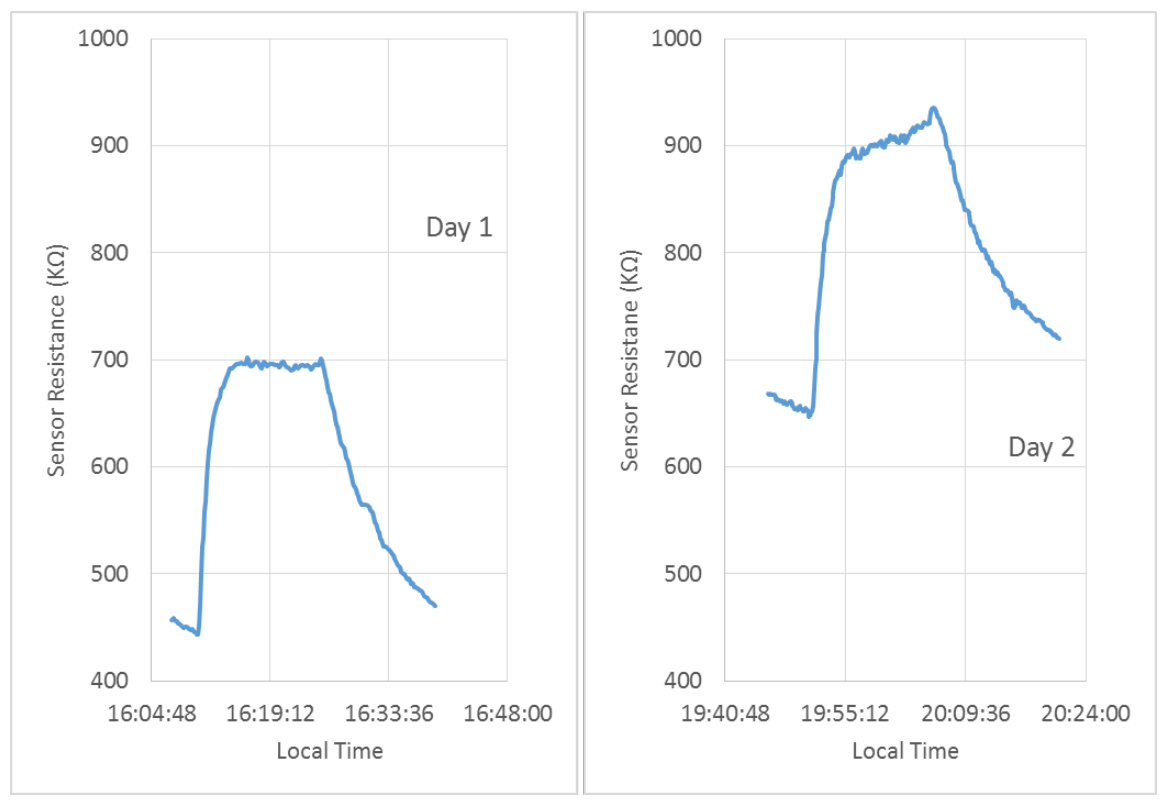

Fig. 7: Acquired time signals of the sensor when exposed to odor coming from stoma pouch in two consecutive days (day 1 , left; day 2, right). The sensor is sensitive to the odor, although the amplitude and baseline of the signal changes due to uncontrolled environmental conditions and drift.

We have obtained high classification performance for stoma pouch odor with the LDA calibration models. After double-cross validation process, once all the samples were left once for test, only one sample was not correctly detected (96\% sensitivity), with no false detections ( $100 \%$ specificity). However, water and urine samples were mixed up. Hence, the developed sensor coupled to a simple classification model is able to detect leaks in the stoma pouch. The system shows limited performance in the discrimination between water and urine ( $72 \%$ of classification rate) due to the difficulty of the task and the similar chemical signature of the samples. However, the discrimination of water and urine does not seem a limiting factor in the envisioned application for stoma pouches, especially since we have been able to show that separation is indeed possible [4]. Figure 8 shows the calibration data and test data for one of the iterations. One can confirm the ability of the model to correctly classify stoma pouch human waste with respect the other two types of samples. Figure 8 also shows that train and test data show similar distributions in the space, confirming thereby the generalization capability for the calibration models. 


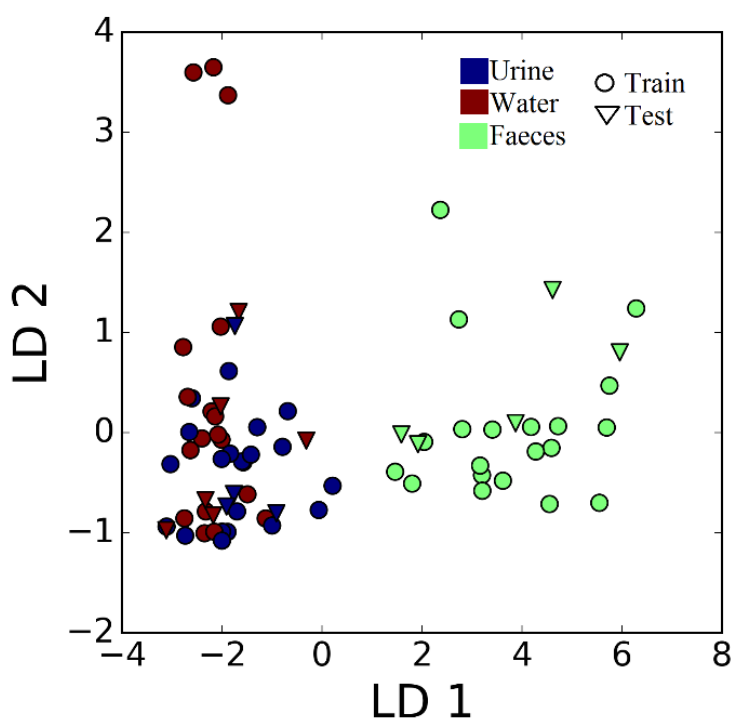

Fig. 8: Example of a calibration model trained with $80 \%$ of the samples and tested with the remaining $20 \%$ of the samples. In this example, the model is able to successfully separate human faces (green) from urine (blue) and water (red). Calibration (circle) and test (triangle) data show similar distribution, confirming the generality of the model.

\section{Conclusion}

In this contribution we have presented a novel method to incorporate $\mathrm{CuO}$ nanospheres into a matrix of nanoporous $\mathrm{ZnO}$. By combining a colloidal suspension with a sol-gel based material we achieve an inkjet printable ink that results in a heteromaterial featuring a $p$-n junction. The approach allows for producing functional layers with well-defined thickness on arbitrary structures. Here, we have used a low-power consuming MEMS platform to demonstrate that the integration of advanced nanomaterials into microtechnology is feasible using industry-ready technologies. The resulting sensor is capable of detecting odors emitted by a leaking stoma pouch and can consequently be used as basis for a system that inconspicuously notifies affected people and help maintain their social inclusion. 


\section{Acknowledgements}

This work was supported by a grant from the Ministry of Science, Research and the Arts of BadenWürttemberg, a grant from the German Federal ministry of Food and Agriculture (BMVL) under grant number 22017014 (IN²Biogas), and the Georg H. Endress foundation. The authors wish to thank Dr. Thilo Ludwig for XRD measurement and Dr. Arno Feist for performing the SEM characterization. S.P. acknowledges funding from the Community of Madrid under grant number 2016-T1/AMB-1695. J.F. acknowledges the support from the Serra Húnter program.

\section{Conflict of Interest statement}

On behalf of all authors, the corresponding author states that there is no conflict of interest.

\section{Reference:}

[1] R. L. Siegel, K. D. Miller, and A. Jemal, “Cancer statistics,” CA Cancer J Clin, vol. 66, no. 1, pp. 730, 2016.

[2] S. M. Vonk-Klaassen, H. M. de Vocht, M. E. M. den Ouden, E. H. Eddes, and M. J. Schuurmans, "Ostomy-related problems and their impact on quality of life of colorectal cancer ostomates: a systematic review," Qual. Life Res., vol. 25, no. 1, pp. 125-133, 2016.

[3] G. Hubbard et al., "Research priorities about stoma-related quality of life from the perspective of people with a stoma: A pilot survey," Heal. Expect., vol. 20, no. 6, pp. 1421-1427, 2017.

[4] A. Ortiz Pérez et al., "Odor-Sensing System to Support Social Participation of People Suffering from Incontinence," Sensors, vol. 17, no. 1, p. 58, 2016.

[5] H. Sato, H. Morimatsu, T. Kimura, Y. Moriyama, T. Yamashita, and Y. Nakashima, "Analysis of 
Malodorous Substances of Human Feces," J. Heal. Sci., vol. 48, no. 2, pp. 179-185, 2002.

[6] J. Kneer, S. Knobelspies, B. Bierer, J. Wöllenstein, and S. Palzer, "New method to selectively determine hydrogen sulfide concentrations using CuO layers," Sensors Actuators, B Chem., vol. 222, pp. 625-631, 2016.

[7] J. Kneer, J. Wöllenstein, and S. Palzer, "Specific, trace gas induced phase transition in copper(II)oxide for highly selective gas sensing," Appl. Phys. Lett., vol. 105, no. 7, p. 073509, Aug. 2014.

[8] S. Knobelspies, B. Bierer, A. Ortiz Perez, J. Wöllenstein, J. Kneer, and S. Palzer, "Low-cost gas sensing system for the reliable and precise measurement of methane, carbon dioxide and hydrogen sulfide in natural gas and biomethane," Sensors Actuators, B Chem., vol. 236, pp. 885-892, 2016.

[9] J. Kneer, J. Wöllenstein, and S. Palzer, "Manipulating the gas-surface interaction between copper(II) oxide and mono-nitrogen oxides using temperature," Sensors Actuators, B Chem., vol. 229, no. 2, pp. 57-62, 2016.

[10] K. Henzler et al., "Investigation of reactions between trace gases and functional CuO nanospheres and octahedrons using NEXAFS-TXM imaging," Sci. Rep., vol. 5, p. 17729, 2015.

[11] E. Comini, C. Baratto, G. Faglia, M. Ferroni, and G. Sberveglieri, "Single crystal ZnO nanowires as optical and conductometric chemical sensor," J. Phys. D. Appl. Phys., vol. 40, no. 23, pp. 7255-7259, 2007.

[12] M. W. Ahn et al., "Gas sensing properties of defect-controlled ZnO-nanowire gas sensor," Appl. Phys. Lett., vol. 93, no. 26, pp. 1-4, 2008.

[13] P. Rai and Y. T. Yu, "Citrate-assisted hydrothermal synthesis of single crystalline ZnO nanoparticles for gas sensor application," Sensors Actuators, B Chem., vol. 173, no. 2, pp. 5865, 2012. 
[14] R. Kumar, O. Al-Dossary, G. Kumar, and A. Umar, "Zinc oxide nanostructures for NO2 gassensor applications: A review," Nano-Micro Lett., vol. 7, no. 2, pp. 97-120, 2015.

[15] M. Hjiri, L. El Mir, S. Leonardi, N. Donato, and G. Neri, “CO and NO2 Selective Monitoring by ZnO-Based Sensors," Nanomaterials, vol. 3, no. 3, pp. 357-369, 2013.

[16] J. Kneer, S. Knobelspies, B. Bierer, J. Wöllenstein, and S. Palzer, "New method to selectively determine hydrogen sulfide concentrations using CuO layers," Sensors Actuators, B Chem., vol. 222, 2016.

[17] Y. Nakamura, "Selective CO Gas Sensing Mechanism with CuO/ZnO Heterocontact," J. Electrochem. Soc., vol. 137, no. 3, p. 940, 1990.

[18] M. Mansournia and L. Ghaderi, “CuO@ZnO core-shell nanocomposites: Novel hydrothermal synthesis and enhancement in photocatalytic property," J. Alloys Compd., vol. 691, pp. 171177, 2017.

[19] B. Li and Y. Wang, "Facile synthesis and photocatalytic activity of ZnO-CuO nanocomposite," Superlattices Microstruct., vol. 47, no. 5, pp. 615-623, 2010.

[20] M. Poloju, N. Jayababu, and M. V. Ramana Reddy, "Improved gas sensing performance of Al doped $\mathrm{ZnO} / \mathrm{CuO}$ nanocomposite based ammonia gas sensor," Mater. Sci. Eng. B Solid-State Mater. Adv. Technol., vol. 227, no. September 2017, pp. 61-67, 2018.

[21] J. X. Wang et al., "Free-standing ZnO-CuO composite nanowire array films and their gas sensing properties," Nanotechnology, vol. 22, no. 32, 2011.

[22] N. M. Vuong, N. D. Chinh, B. T. Huy, and Y. I. Lee, "CuO-decorated ZnO hierarchical nanostructures as efficient and established sensing materials for H2S Gas Sensors," Sci. Rep., vol. 6, no. May, 2016.

[23] J. Kim, W. Kim, and K. Yong, "CuO/ZnO Heterostructured Nanorods: Photochemical Synthesis and the Mechanism of H2S Gas Sensing," J. Phys. Chem. C, vol. 116, no. 29, pp. 15682-15691, 
2012.

[24] R. M. Allaf and L. J. Hope-Weeks, "Synthesis of $\mathrm{ZnO}-\mathrm{CuO}$ nanocomposite Aerogels by the solgel route," J. Nanomater., vol. 2014, no. li, 2014.

[25] K. J. Saji, S. Populoh, A. N. Tiwari, and Y. E. Romanyuk, "Design of p-CuO/n-ZnO heterojunctions by rf magnetron sputtering," Phys. Status Solidi Appl. Mater. Sci., vol. 210, no. 7, pp. 1386-1391, 2013.

[26] G. Nagaraju, Y. Hwan Ko, and J. Su Yu, "Facile synthesis of $\mathrm{ZnO} / \mathrm{CuO}$ nanostructures on cellulose paper and their p-n junction properties," Mater. Lett., vol. 116, pp. 64-67, 2014.

[27] S. Aygün and D. Cann, "Hydrogen sensitivity of doped CuO/ZnO heterocontact sensors," Sensors Actuators, B Chem., vol. 106, no. 2, pp. 837-842, 2005.

[28] Q. Simon et al., "CuO/ZnO nanocomposite gas sensors developed by a plasma-assisted route," ChemPhysChem, vol. 13, no. 9, pp. 2342-2348, 2012.

[29] B. Behera and S. Chandra, "An innovative gas sensor incorporating ZnO-CuO nanoflakes in planar MEMS technology," Sensors Actuators, B Chem., vol. 229, pp. 414-424, 2016.

[30] H. Gao, H. Jia, B. Bierer, J. Wöllenstein, Y. Lu, and S. Palzer, "Scalable gas sensors fabrication to integrate metal oxide nanoparticles with well-defined shape and size," Sensors Actuators $B$ Chem., vol. 249, pp. 639-646, Oct. 2017.

[31] H. Jia et al., "Cu<inf >2</inf>0@ @ PNIPAM core-shell microgels as novel inkjet materials for the preparation of $\mathrm{CuO}$ hollow porous nanocubes gas sensing layers," J. Mater. Chem. C, vol. 6, no. $27,2018$.

[32] M. Gao, L. Li, and Y. Song, "Inkjet printing wearable electronic devices," J. Mater. Chem. C, vol. 5, no. 12, pp. 2971-2993, 2017.

[33] P. Calvert, "Inkjet printing for materials and devices," Chem. Mater., vol. 13, no. 10, pp. 3299$3305,2001$. 
[34] P. Walden, J. Kneer, S. Knobelspies, W. Kronast, U. Mescheder, and S. Palzer, “Micromachined Hotplate Platform for the Investigation of Ink-Jet Printed, Functionalized Metal Oxide Nanoparticles," J. Microelectromechanical Syst., vol. 24, no. 5, pp. 1384-1390, 2015.

[35] H. Gao, J. Amann, X. Lyu, J. Wollenstein, and S. Palzer, "Novel Method for Thermal Characterization of MEMS," J. Microelectromechanical Syst., vol. 27, no. 3, 2018.

[36] R. Viswanatha et al., "Synthesis and Characterization of Mn-Doped ZnO Nanocrystals," 2004.

[37] J. Kneer, A. Eberhardt, P. Walden, A. Ortiz Pérez, J. Wöllenstein, and S. Palzer, “Apparatus to characterize gas sensor response under real-world conditions in the lab," Rev. Sci. Instrum., vol. 85, no. 5, 2014.

[38] R. Gutierrez-Osuna, "Pattern analysis for machine olfaction: a review," IEEE Sens. J., vol. 2, no. 3, pp. 189-202, 2002.

[39] S. Marco and A. Gutierrez-Galvez, "Signal and data processing for machine olfaction and chemical sensing: A review," IEEE Sens. J., vol. 12, no. 11, pp. 3189-3214, 2012.

[40] M. K. Muezzinoglu et al., "Acceleration of chemo-sensory information processing using transient features," Sensors Actuators, B Chem., vol. 137, no. 2, pp. 507-512, 2009.

[41] U. Holzwarth and N. Gibson, "The Scherrer equation versus the 'Debye - Scherrer equation ,'” Nat. Nanotechnol., vol. 6, no. 9, p. 534, 2011.

[42] A. Vergara, S. Vembu, T. Ayhan, M. A. Ryan, M. L. Homer, and R. Huerta, "Chemical gas sensor drift compensation using classifier ensembles," Sensors Actuators, B Chem., vol. 166-167, pp. 320-329, 2012.

[43] A. Ziyatdinov, S. Marco, A. Chaudry, K. Persaud, P. Caminal, and A. Perera, "Drift compensation of gas sensor array data by common principal component analysis," Sensors Actuators, B Chem., vol. 146, no. 2, pp. 460-465, 2010.

[44] R. Huerta, T. Mosqueiro, J. Fonollosa, N. F. Rulkov, and I. Rodriguez-Lujan, “Online 
decorrelation of humidity and temperature in chemical sensors for continuous monitoring," Chemom. Intell. Lab. Syst., vol. 157, pp. 169-176, 2016.

[45] S. De Vito et al., "Sensors and Actuators B : Chemical Calibrating chemical multisensory devices for real world applications : An in-depth comparison of quantitative machine learning approaches," Sensors Actuators B. Chem., vol. 255, pp. 1191-1210, 2018. 